\title{
Assessment of uterine cavity after hystroscopic removal of sub- mucous fibroids by morcellation
}

\author{
Waleed A. Ayad \\ Department of Obstetrics and Gynecology, Al-Azhar Faculty of Medicine, New-Damietta- Egypt \\ Email: waled.ayad@yahoo.com
}

\begin{abstract}
Study Objective: sub- mucous fibroid can be removed hystroscopically by different techniques , hystoscopic shaver represent one of the most recent treatment of intra cavitary fibroids, althought its usage is simple and has a good result for removal of fibroid, it may be complicated by formation of intra uterine adhesions.

Study Objective: to determine the incidence and types of uterine syechia following hystroscopic myomectomy by morcellation. Design: prospective observational study. Patients and methods: Setting: Al- Azhar University hospital (New Damietta) during the period from August 2016 to March 2018. Patients: fifty women who had hystroscopic myomectomy by intra uterine Bigatti shaver (IBS).for different reasons (abnormal uterine bleeding, infertility, recurrent pregnancy loss). Interventions: office hysteroscopy after 2 months from hystroscopic myomectomy by Bigatti shaver. Measurements and Main Results: Evaluation of uterine cavity especially formation of adhesions is observed. The incidence of adhesions was $8 \%$. The development of adhesion was usually associated with multiple myomas present in posterior position who need long resection time and usually the resection is incomplete. The adhesions were usually mild $(75 \%)$ and there was no complications were reported after hysteroscopic myomectomy. Conclusion: the IBS proves to be an effective and safe new instrument for the removal of submucous fibroids, however such procedure may be followed by formation of intra uterine adhesions however most adhesions are filmy and its incidence is less than conventional hystroscopic methods as reviewed in literature.
\end{abstract}

Keywords: hysteroscopy; myomectomy; uterine adhesions; synechiae; integrated Bigatti Shaver.

\section{INTRODUCTION}

Intra uterine morcellator or shaver represents a new alternative technique for removal of uterine fibroids without usage of electricity ${ }^{1}$.

The $1^{\text {st }}$ hystroscopic morcellator was Truclear which was approved by food and drug administration (FDA) in 2005, followed by Myosure tissue removal system in $2009^{2}$.

Intrauterine Bigatti shaver follows the team of hysreoscopic morcellation and has shown to be better than conventional methods ${ }^{3}$.

Formation of Intra Uterine Adhesions (IUA) after hystroscopic surgery found to be $2.4 \%$ of patients undergoing hysteroscopic myomectomy. However, some reports demonstrated a frequent occurrence of IUA which could be as high as $31.3-45.5 \%{ }^{4}$.

Intra uterine adhesions are the most common drawbacks after hystroscopic surgery, and its prevalence is correlated to intra uterine lesions (fibroids, polyps or adhesions) ${ }^{5}$.

In addition to abnormal menses, infertility and recurrent spontaneous abortion are common complaints of IUA, and the accompanying retrograde menstruation may lead to endometriosis. Adhesions are a significant source of impaired organ functioning, decreased fertility, bowel obstruction, difficult reoperation, and possibly, pain with consequent financial sequelae ${ }^{6}$.

\section{PATIENTS AND METHODS}

The present study is a prospective follow up study. The study included 50 women indicated for hystroscopic myomectomy using intrauterine Bigatti shaver (IBS). They were selected from Obstetrics and Gynecology Department, (Al-Azhar Faculty of Medicine, New Damietta), during the period from August 2016 to March 2018.

After full explanation of the study protocol for each patient, an informed consent was signed by each participant.

In addition, the study protocol was approved by the Research and Local Ethics Committee of Al-Azhar Faculty of Medicine.

Inclusion criteria: Patients underwent 
hysteroscopic myomectomy by intrauterine Bigatti shaver (IBS), either G0, G1, G2 with myoma size less than $3 \mathrm{~cm}$.

Exclusion criteria: Suspect

of

malignancy, Active pelvic inflammatory disease (PID), Suspect of pregnancy, Severe vaginal infection, Patient with bleeding tendency and Cardiac patient and patient taking anticoagulant drugs..

Included females in the present work, were submitted to:

Complete history taking including: personal history, menstrual history, obstetric history or history of medical disease such as diabetes or hypertension.

General clinical examination.

Investigations as routine Lab.

Transvaginal sonography.

Hysteroscopic Techniques:

We performed all operations using the intra uterine Bigatti shaver (IBS ) which formed of Rt . Angle telescope with built in inner sheath which contain pathway for metallic shaver which inserted into hand piece (Drill cut- $\mathrm{x}$ Karl Storz GmbH of Tuttlingen) and finally attached to unidrive unit (Unidrive ${ }^{\circledR}$ eco Karl Storz GmbH of Tuttlingen) which controlled by foot switch .The outer sheath is double flow for suction irrigation and its diameter is $24 \mathrm{Fr}$. We used 400 to 500 oscillating rotation power per minute. For irrigation, we used a normal isotonic solution like $0.9 \%$ sodium chloride. The maximum flow setting was 400 $\mathrm{ml} / \mathrm{min}$ with an intrauterine pressure less than $70-90 \mathrm{mmHg}$. Once the pathological site was visualized, we introduced the rigid shaving system connected to the motor drive unit and the roller pump into the operative channel and started the procedure. The duration of the procedure and fluid defect was calculated in each procedure.

Any complications were documented. All patients took $200 \mathrm{mcg}$ misoprostol sublingual $6 \mathrm{hr}$ at least before the procedure. We gave $200 \mathrm{mcg}$ misoprostol sublingual $6 \mathrm{hr}$ before the procedure and all patients took $1 \mathrm{gm} 1^{\text {st }}$ generation cephalosporin. After finishing the procedure intra uterine folly's catheter inflated with $15-20 \mathrm{ml}$ saline is lifted for $8 \mathrm{hr}$.

Then, an outpatient hysteroscopy was routinely performed 2 months after myomectomy to diagnose postoperative uterine adhesion and assess the adhesion score according to the American Fertility Society recommendations. When identified, a $30^{\circ}$ forward-oblique telescope was used to lyse them during the same procedure. For those with any residual myoma, a second and sometimes a third operative hysteroscopy were recommended. Each followed by a postoperative diagnostic hysteroscopy 2 months later.

Patients were classified into two groups; with synechiae (according to classification of American Fertility and Sterility Association) and without synechiae.

Statistical analysis: the collected data were organized, tabulated and analyzed using statistical package for social science (SPSS) version 20 (IBM®SPSS $\AA$, Chicago, USA). The numerical variables were expressed as mean and standard deviation (SD), while categorical variables were presented as number and percentages. Comparison between groups was done by independent samples student (t) test and chi square for qualitative variables. $\mathrm{P}$ value $<0.05$ was considered significant.

\section{RESULTS}

The present study included 50 females who underwent hysteroscopic myomectomy by IBS $®$ shaver. Two months after hysteroscopy, adhesions were developed in 4 patients $(8.0 \%)$. Patients" age ranged from 24 to 36 years; and there was non-significant decrease of age in females with adhesions when compared to those who had no adhesions ( $29.50 \pm 5.44$ vs $31.21 \pm 3.85$ years respectively) (Table 1).

The AP diameter ranged from 4 to $6.5 \mathrm{~cm}$, while fundal isthmic length ranged from 5.40 to $7.50 \mathrm{~cm}$, and finally endometrial thickness ranged from 7 to $13 \mathrm{~mm}$; and there was no significant difference between adhesion and non-adhesion groups. The indication of myomectomy was infertility in $36 \%$, abnormal uterine bleeding (AUB) in $38 \%$ and pelvic pain in $26 \%$ and there was no significant difference between adhesion and non-adhesion groups. The number of myoma in studied females was single in 45 females (90.0\%) and multiple in 5 females $(10.0 \%)$ and there was statistically significant increase of patients with multiple myomas in adhesion group when compared to non-adhesion group (50.0\% vs $6.5 \%$ respectively). The site of 
myoma was anterior in $44 \%$, posterior in $10 \%$ and fundal in $46 \%$ and there statistically significant ice of posterior position in group with adhesion when compared to nonadhesion group (75.0\% vs $4.3 \%$ respectively). Myoma grade was G0 in $22 \%$, G1 in $20.0 \%$ and $\mathrm{G} 2$ in $58.0 \%$ and there was no significant difference between group with adhesions when compared to non-adhesion group. The myoma size ranged from 1.5 to 3 and there was no significant difference between adhesion and non-adhesion groups (Table 1).

As regard to cervical dilatation time, it ranged from 1 to 3 minutes, while total operative time ranged from 24 to 38 minutes; and there was no significant difference between adhesion and non-adhesion groups. On the other hand, the morcellation time ranged from 14 to 23 minutes and there was statistically significant increase of morcellation time in adhesion when compared to non-adhesion groups $(20.25 \pm 0.95$ vs $17.65 \pm 1.82$ minutes respectively). The amount of fluids used during hysteroscopy ranged from 2000 to $3750 \mathrm{cc}$, and there was no significant difference between adhesion and nonadhesion groups. Myoma was removed completely in $96 \%$ of all studied females and incomplete in $4 \%$. All cases in non-adhesion group had complete resection, while $50 \%$ of females with adhesion had complete resection. Thus, there was significant increase of incomplete removal in adhesion when compared to non-adhesion group $(50.0 \%$ vs $0.0 \%$ respectively). Duration of hospital stay after the hysteroscopy, it ranged from 3 to 8 hours; and there was no significant difference between both adhesion and non-adhesion groups. The grade of adhesion was mild in 3 out of 4 females $(75.0 \%)$ and moderate in only one female (25.0\%) (Table 2 ).

No complications were reported in the present work.

Table (1): patient age, ultrasound data and myoma characteristics among studied females

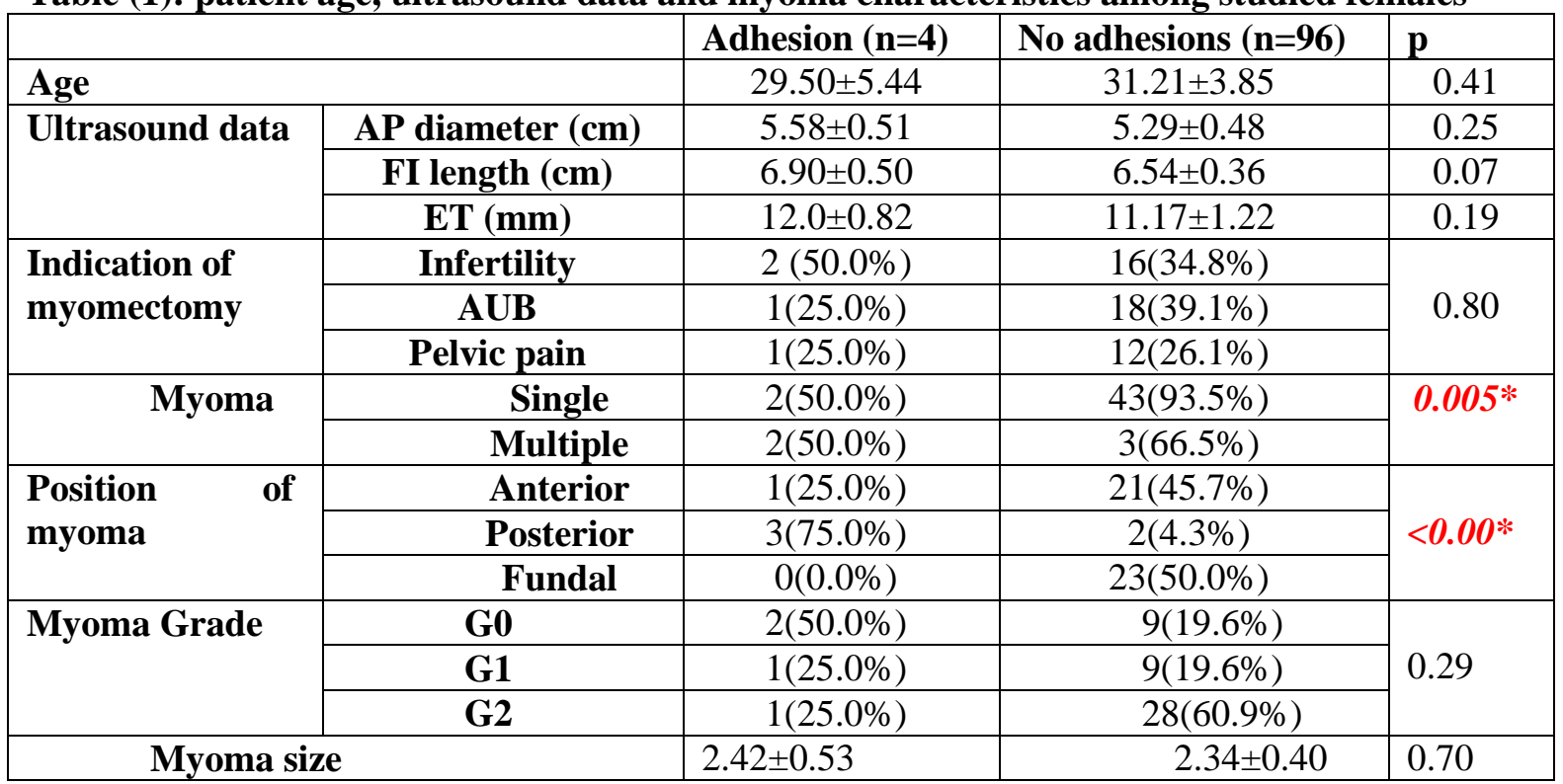

AP: anteroposterior; FI: fundal-isthmic; ET: endometrial thickness; *: Significant difference.

Table (2): Operative data among studied females

\begin{tabular}{|c|c|c|c|c|}
\hline \multicolumn{2}{|c|}{ the } & Adhesion $(n=4)$ & No adhesions (n=96) & $\mathbf{p}$ \\
\hline \multicolumn{2}{|c|}{ Cervical dilatation time (min) } & $1.25 \pm 0.50$ & $1.32 \pm 0.52$ & 0.77 \\
\hline \multicolumn{2}{|c|}{ Operative time (min) } & $34.25 \pm 5.566$ & $32.95 \pm 3.14$ & 0.46 \\
\hline \multicolumn{2}{|c|}{ Morcellation time (min) } & $\begin{array}{l}20.25 \pm 0.9 \\
5\end{array}$ & $17.65 \pm 1.82$ & $0.008 *$ \\
\hline \multicolumn{2}{|c|}{ Amount of fluid } & $3062.50 \pm 773.92$ & $2940.21 \pm 391.52$ & 0.58 \\
\hline \multirow{2}{*}{$\begin{array}{l}\text { Myoma } \\
\text { Resection }\end{array}$} & Complete & $2(50.0 \%)$ & $46(100.0 \%)$ & \multirow[t]{2}{*}{$<0.001 *$} \\
\hline & Incomplete & $2(50.0 \%)$ & $0(0.0 \%)$ & \\
\hline \multicolumn{2}{|c|}{ Duration of hospital stay } & $5.25 \pm 1.89$ & $4.91 \pm 0.89$ & 0.51 \\
\hline \multirow{2}{*}{$\begin{array}{l}\text { Grade of } \\
\text { adhesion }\end{array}$} & Mild & $3(75.0 \%)$ & - & \\
\hline & Moderate & $1(25.0 \%)$ & - & \\
\hline
\end{tabular}


Assysment of uterine cavity after hystroscopic removal of sub- mucous fibroids by morcellation

*: Significant difference.

\section{DISCUSSION}

The present study was designed to evaluate uterine cavity specially formation of adhesion after hysteroscopic myomectomy. It was carried out at the Department of Obstetrics and Gynecology, Al-Azahr University hospital (New Damietta). It included 50 females who underwent hysteroscopic myomectomy for different causes using the intrauterine Bigatti Shaver IBS, and reviewed for intrauterine adhesions 2 months after the primary procedure.

As regard to indication of myomectomy, it was infertility in $36 \%$, abnormal uterine bleeding (AUB) in $38 \%$ and pelvic pain in $26 \%$ and there was no significant difference between adhesion and non-adhesion groups. These results going in agreement with previous studies showed that, the most common clinical presentation symptoms of uterine myomas are bleeding, secondary anemia due to menometrorrhagia, metrorrhagia or intermenstrual bleeding, pain, sexual dysfunction, and infertility. More than just one symptom is present in up to $62 \%$ of the affected women. In addition, Bhandari $\boldsymbol{e t}$ $\boldsymbol{a l}{ }^{7}$. reported that, most of the participants $(72.55 \%)$ were cases of primary infertility.

In their study, Bahar et $^{\boldsymbol{a l}^{8}}{ }^{\mathbf{8}}$. reported that, the most prevalent initial symptoms were irregular uterine bleeding $(35.5 \%)$ and infertility (21.9\%). About $20 \%$ of women had no symptoms and underwent a hysteroscopic procedure because of an abnormal finding at imaging during the routine annual check-up. This is quietly different from the present work and can be explained by different inclusion criteria. In addition, Yendru et al. ${ }^{9}$ reported that, females with uterine myomas presented with different symptoms, like abnormal uterine bleeding (AUB), infertility and recurrent pregnancy loss (RPL)

The high safety of IBS is explained by the following facts: it is able to remove the tissue chips at the same time as resection so that the procedure, always done under visual control, becomes faster, very precise, easier to perform, and with fewer complications

A randomized prospective study published by Bigatti et al.$^{\mathbf{1 0}}$ showed that especially for the treatment of large polyps and myomas up to 2 $\mathrm{cm}$ it has several well-described advantags. As both operating and resection times were statistically significantly in favor of the IBS. No bleeding or major complication was observed in the IBS group. Not only operation time but also total fluid loss appeared to be better with the IBS ${ }^{\circledR}$ system. In fact, this study demonstrated that using the IBS for myoma resection, the median total operating time was 23.25 min with a medium resection time of $15.08 \mathrm{~min}$. In addition, the main advantage of the IBS was that the myomas were effectively enucleated from their fovea, and the intramural site of insertion of the myoma was removed. The surrounding healthy endometrium was avoided without any thermal injury occurring compared with the less precise behavior of conventional resectoscopy. No coagulation was needed, and there were no excess bleeding problems.

Yang et al.$^{11}$ showed that the intrauterine Bigatti shaver is a safe and effective method for resection of endometrial polyps. The continuous flow of fluid and suction of tissue fragments offers a definite advantage by providing constant clear visualization. This is crucial in hysteroscopic surgery as an obscured operating field is the main reason for serious complications such as uterine perforation and prolonged surgery leading to fluid overload.

No reports were found for post-IBS uterine adhesion in literature. Here, the incidence of adhesion post-hysteroscopic myomectomies were reported to be $8 \%$ which is significantly lower than conventional resectoscopy, the adhesion formation is one of the most common complications of hystero-scopic myomectomy reported up to $31.3 \%$ in a single myoma and $45.5 \%$ in multiple myoma as reported by Deans and Abbott ${ }^{12}$. Results of the present work, confirmed that, the posthysteroscopic adhesions is associated with multiplicity of myomas, as there was statistically significant increase of patients with multiple myomas in adhesion group when compared to non-adhesion group (50.0\% vs $6.5 \%$ respectively). 
Shokeir et al. ${ }^{13}$ reported a $60 \%$ rate of intrauterine adhesions with degree I, $10 \%$ with degree II, and 5\% with degree III, 2-4 weeks after resectoscopic procedures for different endocavitary pathologies. Even though the study considered all hysteroscopic procedures and no detailed information was available regarding the prevalence after myomectomy, only $25 \%$ of the studied population showed an anatomically uninjured uterine cavity. A later follow-up evaluation at 12 months showed a significant difference in distribution, with a prevalence of vascular adherences compared to filmy synechiae. In addition, Touboul et al. ${ }^{14}$ reported an incidence of $7.5 \%$ with bipolar resection, while resection with monopolar electrodes was reported to be $35-45 \%$. They attributed the low rate of synechiae to the use of bipolar energy, considering it safer, less prone to accidental electric fluxes, and therefore not damaging the healthy myometrium.

In many studies, the rate of intrauterine adhesions is variable from $1.07 \%$ to $78 \%$ depending on the use of electrocautery and adhesion preventive strategies. The reported rate in the present work $(8.0 \%)$ lies within the reported rate in literature. On the other hand, Mazzon et al. ${ }^{15}$ reported that, in 29 patients (4.23\%), synechiae of the uterine cavity after resectoscopic surgery was found. This value is much lower than the present work, and it may be attributed to different inclusion criteria, where they included all myomas regardless of its size and included all females regardless of their age group.

As regard resection in the present work, it was complete in $96 \%$ of all studied females and incomplete in $4 \%$. All cases in non-adhesion group had complete resection, while $50 \%$ of females with adhesion had complete resection. Thus, there was significant increase of incomplete removal in adhesion when compared to non-adhesion group $(50.0 \%$ vs $0.0 \%$ respectively). Mazzon et al. ${ }^{15}$ reported that, the persistence in the uterine cavity of myomas that were only partially removed during surgery (residue myoma) may play a role in the development of long-term adhesions. The traumatized residual portion of the myoma in contact with the endometrium of the opposite side may facilitate the formation of columnar fibrous adherences. Therefore, a follow-up evaluation after a hystroscopic procedure is important for evaluating the complete removal of a myoma with a new surgical treatment. In their study, during the follow-up phase, new myomas or residual myomas were detected in 56 patients $(8.17 \%)$ (12\% in the present study).

In the present work, there was no significant association between the size of fibroid and incidence of postoperative intrauterine adhesions. These results are comparable to those reported by Asgari et al. ${ }^{16}$ who found a non-significant relation between the two.

The grade of adhesion in the present work was mild in 3 out of 4 females $(75.0 \%)$ and moderate in only one female (25.0\%). Bhandari et al. ${ }^{7}$ reported that, of the 51 patients underwent myomectomy, intrauterine adhesions were observed in consequent hysteroscopy in 11 cases $(21.57 \%)$. They added, of these 11 adhesion cases, mild adhesions were noted in seven cases, while moderate adhesions were present in three cases. Severe type of adhesion was observed in only one patient. The higher rate of adhesion in their work can be explained by the fact that, they included all myoma types and sizes, and the presence of multiple myomas in their study.

\section{Conclusion}

The IBS proves to be an effective and safe new instrument for the removal of submucous fibroids, however such procedure may be followed by formation of intra uterine adhesions however most adhesions are filmy and its incidence is less than conventional hystroscopic methods as reviewed in literature. It is also proposed that, electrical current-free resection of myomas could significantly reduce the postoperative adhesions formation. However, due to small number of patients included in the present study, future trials on wide range of patients are recommend to confirm this proposal.

\section{REFERENCES}

1-Emanuel MH and Wamsteker K (2005):

The intrauterine morcellator: a new hysteronscopic operating technique to remove intrauterine polyps and myomas. J Minim Invasive Gynecol., 12(1):65-66. 
Assysment of uterine cavity after hystroscopic removal of sub- mucous fibroids by morcellation

2-Orady ME and Aslanova R (2015): Office Operative Hysteroscopy: Polyp and Submucosal Fibroid Removal. https://link.springer.com/content/pdf/10.1007 \%2F978-1-4939-1414-2.pdf

3-Bigatti G (2011): IBS® Integrated Bigatti Shaver, an alternative approach to operative hysteroscopy. Gynecol Surg., 8(2):187-191.

4-Taskin O, Sadik S, Onoglu $A$ and Gokdeniz R (2000): Role of endometrial suppression on the frequency of intrauterine adhesions after resectoscopic surgery. J Am Assoc Gynecol Laparosc., 7:351-354.

5-Revaux A, Ducarme $G$ and Luton $D$ (2008): Prevention of intrauterine adhesions after hysteroscopic surgery. Gynecol Obstet Fertil., 36:311-317.

6-March CM (2011): Asherman's syndrome. Semin Reprod Med., 29(2):83-94.

. 7-Bhandari S, Ganguly I, Agarwal P, Singh A and Gupta N (2016): Effect of myomectomy on endometrial cavity: A prospective study of 51 cases. J Hum Reprod Sci., 9:107-111

8-Bahar R, Shimonovitz M, Benshushan A and Shushan A. (2013): Case control study of complication associated with bipolar and monopolar hysteroscopic operations. J Min Invasive Gynecol., 20:376-380.

9-Yendru KS, Yelamanchi SD and Vaddiraju GB (2018): Hysteroscopic Resection of Submucous Fibroids in Symptomatic Women. The Journal of Obstetrics and Gynecology of India , https://link.springer.com/article/10.1007/s132 24-018-1139-z
10-Bigatti G, Ferrario C, Rosales M, Baglioni A and Bianchi S.(2012): IBS ${ }^{\circledR}$ Integrated Bigatti Shaver versus conventional bipolar resectoscope: a randomised comparative study. Gynecol Surg., 9(1):6372.

11-Yang L, Rajesh $H$ and Yu S (2017): The Intrauterine Bigatti Shaver for Endometrial Lesions: Experience and Modifications. Open J Obstet Gynecol., 7: 1-6.

12-Deans $R$ and Abbott J (2010): Review of intrauterine adhesions. J Minim Invasive Gynecol., 17(5):555-569.

13-Shokeir TA, Fawzy $M$ and Tatongy $M$ (2008): The nature of intrauterine adhesions following reproductive hysteroscopic surgery as determined by early and late follow-up hysteroscopy: clinical implications. Arch Gynecol Obstet., 277:423-427.

14-Touboul C, Fernandez $H$ and Deffieux $X$ (2009): Uterine synechiae after bipolar hysteroscopic resection of submucosal myomas in patients with infertility. Fertil Steril., 92:1690-1693.

15-Mazzon I, Favilli A and Cocco P (2014): Does cold loop hysteroscopic myomectomy reduce intrauterine adhesions? A retrospective study. Fertil Steril., 101: 294-298.

16-Asgari Z, Hafizi L, Hosseini R, Javaheri $A$ and Rastad H (2015): Intrauterine synechiae after myomectomy; laparotomy versus laparoscopy: Non-randomized interventional trial. Iran J Reprod Med., 13:161-168. 